\title{
Desenvolvimento inicial de plântulas de Theobroma grandiflorum (Willd. ex Spreng.) Schum. sob influência de sombreamento.
}

Rubens Ribeiro da SILVA ${ }^{1}$, Gilson Araújo de FREITAS², Susana Cristine SIEBENEICHLER ${ }^{1}$, Jhansley Ferreira da MATA², Jaíza Ribeiro CHAGAS².

\section{RESUMO}

O presente estudo foi conduzido com o objetivo de avaliar o comportamento inicial de plântulas de Theobroma grandiflorum (Willd. ex Spreng.) Schum. (cupuaçu), em função de diferentes níveis de sombreamento. Ao final de 50 dias, após a emergência, as plântulas de cupuaçu foram submetidas a três níveis de sombreamento, sendo: $0 \%$ de sombreamento, $50 \%$ de sombreamento e Sombra Natural. O crescimento das mudas foi conduzido em delineamento de blocos casualizados, com 15 repetições, sendo cada planta considerada como uma repetição. Foram avaliados a altura, o diâmetro e o número de folhas aos 60, 82, 103, 124, 145 dias após a emergência das plântulas. A Massa Seca de Folhas (MSF), Massa Seca do Caule (MSC), Massa Seca da Raiz (MSR) e Massa Seca Total (MST), Relação parte aérea/raiz (PA/R) e relação Altura da planta/Diâmetro do colo (A/D), foram avaliadas no final do experimento. O crescimento inicial de Theobroma grandiflorum foi corroborado com os padrōes da espécie, que ocorre no interior das matas primárias, tendo melhor desenvolvimento dos parâmetros avaliados em condiçôes de $50 \%$ de sombreamento. A condição de $50 \%$ de sombreamento pode ser recomendada para a formação de mudas de Theobroma grandiflorum, devido o seu melhor desempenho em altura, diâmetro, número de folhas e alocação de massa nas partes da planta.

\section{PALAVRAS-CHAVE}

Nível de sombreamento, Adaptação, Desenvolvimento inicial, Alocação de biomassa.

\section{Initial development of Theobroma grandiflorum (Willd. ex Spreng.) Schum. saplings under shading influence}

\begin{abstract}
The objective of this work was to evaluate the initial growth of sapling of Theobroma grandiflorum (cupuaçú), in function of different levels of solar radiation. Fifty days, after the emergency, the saplings were submitted to three levels of shade, being: $0 \%, 50 \%$ and natural shade. Sapling development was carried out in randomized block design with 15 repetitions (15 saplings). The sapling height, lap diameter and the numbers of leaves being evaluated on the $60^{\text {st }}, 82^{\text {nd }}, 103^{\text {rd }}, 124^{\text {th }}, 145$ days after sapling emergence. The Dry Mass of the leaves (DML), Dry Mass of the stem (DMS), Dry Mass of the root (DMR) and Dry mass of the plant (DMP), Relation shoot/root (PA/R) and relation plant height/Lap diameter, were measured at the end of the experiment, when the sapling reached the appropriate size to be planted in the field. The initial growth of Theobroma grandiflorum was corroborated with the species standard that occurs inside of the primary bushes, so its development of the parameters evaluated is better in conditions of $50 \%$ of shade. The condition of $50 \%$ of shade can be recommended for the formation of plants of Theobroma grandiflorum, which had the better performance in plant height, lap diameter, number and allocation of mass in the parts of the plant.
\end{abstract}

\section{KEYWORDS}

Level of radiation, Adaptation, Initial development, Allocation of biomass.

\footnotetext{
1 Universidade Federal do Tocantins/ Campus Universitário de Gurupi, Rua Badejos Lt. 07 Chácaras 69 e 72. CEP 77402-970 Caixa Postal 66, Gurupi-T0. e-mail: rrs2002@uft.edu.br

2 Acadêmico de agronomia, Universidade Federal do Tocantins. e-mail: freitas@uft.edu.br
} 


\section{INTRODUÇÃO}

A qualidade do ambiente é preocupação mundial e tem se mostrado cada vez mais freqüente nos últimos anos. Isto faz com que ocorra um aumento na demanda de serviços e produtos, em especial a produção de mudas de espécies florestais nativas. A exploração desordenada dos recursos naturais tem gerado a degradação de áreas em quase todo o território nacional (Ferreira, 2000 apud Scalon et al., 2003). Várias pesquisas sobre a propagação, a emergência e o desenvolvimento de plantas nativas têm sido realizadas no Brasil. Entretanto, poucos estudos foram encontrados na literatura sobre a espécie Theobroma grandiflorum (Willd. Ex Spreng.) Schum, espécie nativa da Amazônia, principalmente o estado do Pará.

O cupuaçuzeiro (Theobroma grandiflorum) é uma das mais importantes fruteiras da Amazônia. Ocorre preferencialmente em várzeas férteis não inundáveis no interior de matas primárias. É uma espécie arbórea da família Sterculiaceae, em cultivos comerciais, o porte da planta varia de 4 a $8 \mathrm{~m}$ de altura, podendo atingir até $18 \mathrm{~m}$ de altura, quando encontrada espontaneamente nas áreas de mata do sul e nordeste da Amazônia Oriental e nordeste do Maranhão (Muller, 1995). Os frutos são comestíveis e muito apreciados, principalmente pelas populações no norte do país, sendo cultivado em pomares domésticos e comerciais. Seu florescimento ocorre durante os meses de setembro-novembro. Os frutos são colhidos diretamente no chão, quando iniciarem a queda espontânea (Lorenzi, 2000).

A luz é um dos fatores que influenciam o crescimento dos vegetais, por ser fonte primária de energia relacionada à fotossíntese. Foram realizados estudos sobre o efeito do sombreamento no desenvolvimento de mudas de diversas espécies amazônicas cultivadas em viveiro (Barbosa, 1985; Pinho et al., 1993). Espécies, como o cupuaçu (Theobroma grandiflorum (Willd. Ex Spreng.) Schum), com potencial para uso em programas de reflorestamento ou geração de renda, devem ser estudadas quanto ao seu comportamento em ambientes sombreados, semelhantes ao habitat natural.

Segundo Moraes Neto et al. (2000) essa capacidade das plantas crescerem rapidamente quando sombreadas é um mecanismo importante de adaptação da espécie, o que constitui uma valiosa estratégia para escapar às condições de baixa intensidade luminosa.

O ambiente de luz em que a planta cresce é de fundamental importância, pois a adaptação das plantas a este ambiente depende do ajuste do seu aparelho fotossintético, de modo que a luminosidade ambiente seja utilizada da maneira mais eficiente possível. O sucesso na adaptação de uma espécie a ambientes com baixa ou alta radiação está associado à eficiência na partição dos fotoassimilados para diferentes partes da planta e na rapidez em ajustar variáveis morfofisiológicas no sentido de maximizar a aquisição dos recursos primários (Dias-Filho, 1997). Assim, postula-se que as espécies características de estádios finais de sucessão apresentam um crescimento mais lento em relação às dos estádios iniciais e intermediários (Bazzaz, 1979).

O crescimento das plantas pode refletir a habilidade de adaptação das espécies às condiçōes de radiação do ambiente em que estão se desenvolvendo. Geralmente as características de crescimento são utilizadas para inferir o grau de tolerância ou de intolerância das espécies à baixa disponibilidade de luz (Scalon et al., 2002).

O presente estudo teve como objetivo avaliar o desenvolvimento inicial de plântulas de Theobroma grandiflorum (cupuaçu), em função de diferentes níveis de sombreamento.

\section{MATERIAL E MÉTODOS}

O presente estudo foi conduzido no período de dezembro de 2005 a abril de 2006, na área experimental da Universidade Federal do Tocantins (UFT), Campus Universitário de Gurupi, localizado na regiáo sul do Estado do Tocantins. A altitude da área experimental é de $280 \mathrm{~m}$, e as coordenadas são $11^{\circ} 43^{\prime} 45^{\prime \prime}$ de latitude e $49^{\circ} 04^{\prime} 07^{\prime \prime}$ de longitude.

A classificação climática da área experimental, segundo KÖPPEN (1948), é do tipo B1wA'a' úmido com moderada deficiência hídrica. A temperatura média anual é de $29,5^{\circ} \mathrm{C}$, com precipitação anual média de $1804 \mathrm{~mm}$, sendo um verão chuvoso e um inverno seco.

O experimento foi conduzido em delineamento de blocos ao acaso, com três níveis de sombreamento e 15 repetições, sendo cada planta considerada como uma repetição.

Os frutos de cupuaçu (Theobroma grandiflorum) foram coletados em 26 plantas matrizes selecionadas quanto à sanidade vegetal e produção, em plantios comerciais e nativos no município de São Domingos do Araguaia-PA, no mês de novembro de 2005 (sendo 15 matrizes de plantios comerciais e 11 matrizes de plantas nativas). Após o transporte do fruto para o local do experimento, as sementes foram extraídas do fruto 6 horas antes da implantação do experimento.

Após a retirada das sementes, estas foram colocadas para germinar em canteiro de areia lavada, localizado em viveiro com $50 \%$ da radiação solar. Após germinarem e atingirem um par de folhas, as mudas foram transplantadas para sacos de polietileno com $0,28 \mathrm{~m}$ de altura e $0,15 \mathrm{~m}$ de diâmetro, contendo aproximadamente 2 litros de substrato orgânico (composto orgânico: 2:1:1, sendo 2: terra preta, 1: palha de arroz carbonizada e 1: ingesta bovina do rúmen.

A composição química do substrato utilizado no desenvolvimento das plântulas foi: $\mathrm{pH}\left(\mathrm{CaCl}_{2}\right)$ - 5,2; Matéria Orgânica - 4,7\% ; Cálcio - 3,25 $\mathrm{cmol}_{\mathrm{c}} \mathrm{dm}^{-3}$; Magnésio - 
4,28 $\mathrm{cmol}_{\mathrm{c}} \mathrm{dm}^{-3}$; Alumínio- 0,39 $\mathrm{cmol}_{\mathrm{c}} \mathrm{dm}^{-3}$; Hidrogênio+ Alumínio- 3,67 $\mathrm{cmol} \mathrm{dm}^{-3}$; Potássio- 39,1 $\mathrm{mg} \mathrm{dm}^{-3}$; Fósforo $-14,8 \mathrm{mg} \mathrm{dm}^{-3}$.

Ao final de 50 dias, após a emergência, as mudas de cupuaçu foram submetidas a três níveis de sombreamento, sendo: $0 \%$ de sombreamento, $50 \%$ de sombreamento e Sombra Natural. No tratamento com sombra natural, as mudas foram colocadas em uma área remanescente de vegetação nativa do cerrado Stricto senso, enquanto o nível de $50 \%$ de sombra foi obtido com tela de polietileno de coloração preta (sombrite).

Após dez dias de aclimatação, foram iniciadas as avaliações de altura, diâmetro do colo e número de folhas. Os dados foram coletados no intervalo de 21 dias, nos seguintes períodos de desenvolvimento das plântulas: 60, 82, 103, 124 e 145 dias após a emergência (DAE). Durante as avaliaçōes as mudas foram alocadas eqüidistantes de $0,4 \mathrm{~m}$.

Os atributos usados como indicadores do efeito da luminosidade sobre o desenvolvimento inicial do cupuaçu foram: Altura da Planta (AP), Diâmetro de Colo (DC), Número de Folhas (NF), Massa Seca de Folhas (MSF), Massa Seca do Caule (MSC), Massa Seca da Raiz (MSR) e Massa Seca Total (MST), Relação parte aérea/raiz (PA/R) e relação Altura da planta/Diâmetro do colo (A/D).

Para avaliação de altura de planta e diâmetro do colo, considerou-se $100 \%$ das mudas. Já para as avaliaçôes de massa seca de folhas, caule e raízes foram considerados apenas $30 \%$ das plantas de cada tratamento, tomadas aleatoriamente mediante sorteio, realizadas apenas no final do experimento. A altura do caule foi considerada desde o colo até o ápice de cada planta, medida com régua graduada em centímetros $(\mathrm{cm})$, e o diâmetro do colo medido com paquímetro graduado em milímetros $(\mathrm{mm})$, tomado a $1 \mathrm{~cm}$ do solo.

Para determinação de massa seca de raízes, caule e folhas, o material foi separado (folhas, caule e raízes), sendo o mesmo pesado separadamente em balança semianalítica e acondicionado em sacos de papel devidamente identificados, os quais foram colocados em estufa com circulação de ar forçada, à aproximadamente $70^{\circ} \mathrm{C}$, durante 72 horas.

Os resultados foram submetidos à análise de variância, sendo as médias comparadas pelo teste de Tukey, a nível de $5 \%$ de probabilidade.

\section{RESULTADOS E DISCUSSÃO}

$\mathrm{Na}$ avaliação do desenvolvimento inicial das mudas de cupuaçu através da altura de plantas foi observada diferença estatística em função da variação de luminosidade em todas as datas de coleta, com exceção para os 60 e 82 DAE (Figura 1A). Foi observado que as plantas de cupuaçu ao serem cultivadas em ambiente sob $50 \%$ de sombreamento apresentaram maior crescimento em altura do que as plantas cultivadas em ambiente de $0 \%$ de sombreamento e sombra natural. Esta observação é corroborada pelo seu desenvolvimento no ambiente natural que ocorre no interior das matas primárias (Lorenzi, 2000). Durante o período de avaliação (60 a 145 DAE) das plantas através da altura, foi observado que houve diferença estatística nos três níveis de sombreamento.

$\mathrm{Na}$ avaliação do diâmetro do colo, foi observado que as plantas de cupuaçu apresentaram o mesmo comportamento nos três níveis de sombreamento durante o período estudado (Figura 1B). Com exceção aos $145 \mathrm{DAE}$, onde as submetidas a $50 \%$ de sombreamento apresentaram o diâmetro do colo maior. Resultado semelhante, da resposta do cupuaçuzeiro em ambiente de 50\% de sombreamento, foi encontrado com a espécie Theobroma cacao que pertence à mesma família do cupuaçu (Murray \& Nichols, 1996).

Com relação ao número de folhas, as plantas de cupuaçu cultivadas em ambiente de sombra natural apresentaram menor número de folhas, o que pode ser atribuído à condição de extremo sombreamento, reduzindo as atividades metabólicas da planta. $\mathrm{O}$ maior número de folhas foi encontrado em plantas cultivadas em ambiente sob 50\% de sombreamento, como também, o maior número de folhas proporciona uma maior quantidade de fotoassimilados produzidos, que serão translocados para o crescimento em altura (Figura 1A) e diâmetro do colo (Figura $1 \mathrm{~B}$ ) e para formação de massa seca (Figura 2). Campos \& Uchida (2002), trabalhando com uma espécie nativa da Amazônia (Jacarandá copaia) encontraram maior número de folhas em ambiente sob $30 \%$ de sombreamento. No período estudado, foi observado que a partir dos $103 \mathrm{DAE}$ as plantas submetidas a ambiente de 50\% de sombreamento, tiveram maior tendência de investimento em quantidade de folhas, o que pode ser atribuída à melhor eficiência na translocação de fotoassimilados e metabolismo da planta (Figura 1C).

Quanto à alocação de massa seca nas raízes, caule e folhas em plantas de Theobroma grandiflorum, foi observado o maior investimento em massa seca, tanto para a planta inteira quanto para caule, raízes e folhas nas plantas que estavam na condição de $50 \%$ de sombreamento (Figura 2), Avaliação similar foi encontrada por Felfili et al. (1999) em Sclerolobium paniculatum onde os maiores valores de massa seca de folhas foram observados nas plantas cultivadas em ambiente de 50\% de sombreamento.

No parâmetro massa seca total (MST), o maior investimento foi observado nas plantas expostas em ambiente de $50 \%$ de sombreamento, apresentando diferença daquelas cultivadas em $0 \%$ e $90 \%$. Isso pode ser explicado através da maior eficiência no aproveitamento da incidência luminosa, influenciando em maior produção de fotoassimilados e conseqüentemente maior MST da planta (Figura 2). 

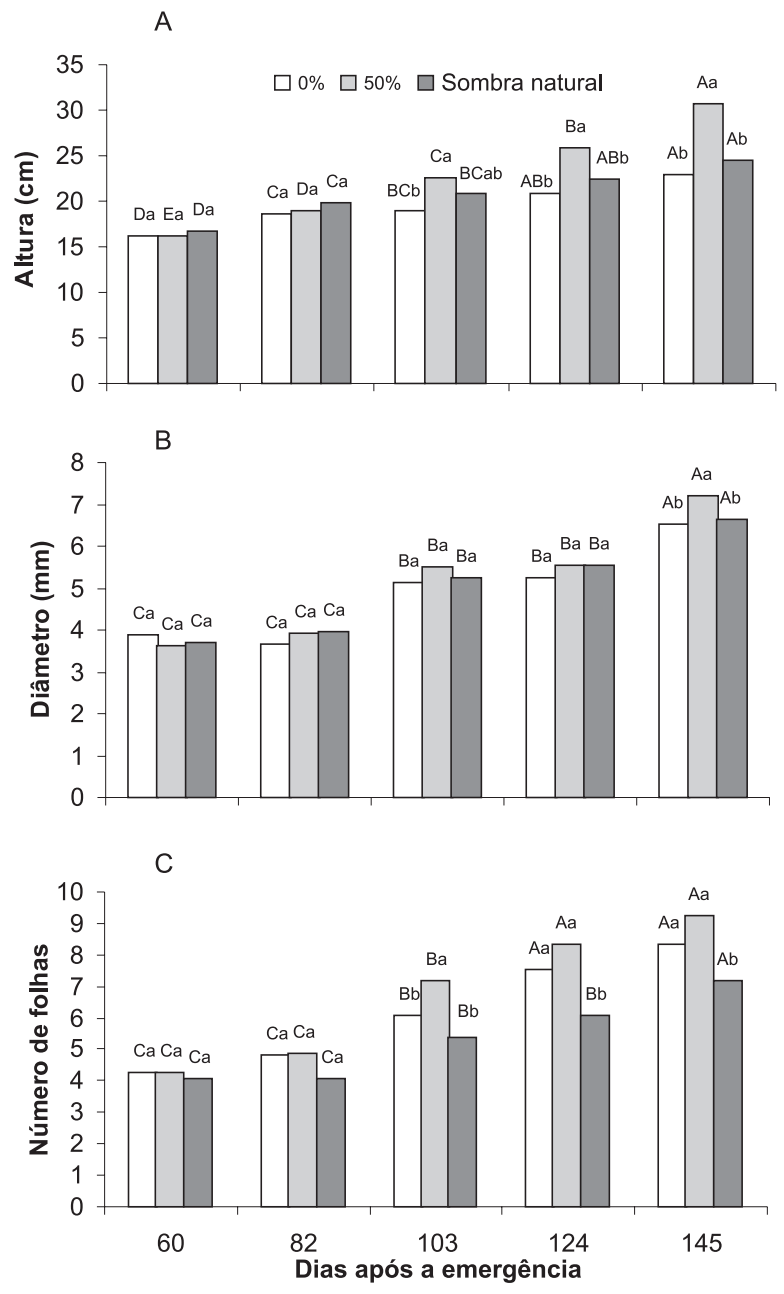

Figura 1 - Altura do caule (cm) (a), diâmetro do colo (mm) (b) e número de folhas (c) de plantas de Theobroma grandiflorum (Willd. Ex Spreng.) Schum, sob três níveis de sombreamento, Gurupi-T0, 2006. Médias seguidas pelas mesmas letras maiúsculas considerando DAE e letras minúsculas considerando os níveis de luz não diferem estatisticamente entre si, a 5\% de probabilidade pelo teste tukey.

Em outras espécies já estudadas, como Muntingia calabura, foi encontrado comportamento semelhante nas mudas cultivadas em condições sombreadas (Castro et al., 1996). No entanto, alguns trabalhos, têm mostrado maiores acúmulos de massa seca total nas plantas cultivadas a pleno sol (Mazzei et al., 1998; Groninger et al., 1996). A resposta de acúmulo de massa seca é variável, devido cada espécie possuir características genéticas diferentes e conseqüentemente, adaptaçōes fisiológicas diferentes.

No parâmetro de relação (PA/R), o maior valor foi encontrado nas plantas em ambiente de sombra natural. Esse aumento na relação $\mathrm{PA} / \mathrm{R}$ ocorreu devido à baixa atividade metabólica nas folhas, reduzindo a produção de

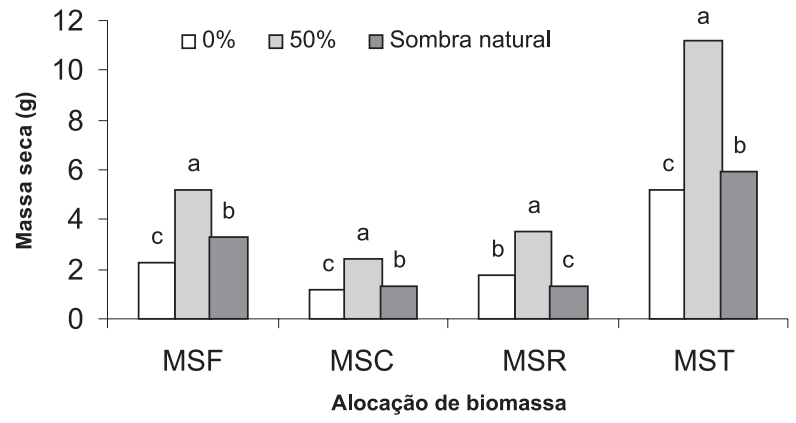

Figura 2 - Distribuição da massa seca nas folhas (MSF), caule (MSC), raízes (MSR) e total (MST) em plântulas de Theobroma grandiflorum (Willd. Ex Spreng.) Schum, aos 145 dias DAE, submetidas a diferentes níveis de sombreamento.

fotoassimilados que seriam translocados para as raízes. Esse baixo investimento em raízes e conseqüente aumento em área foliar também pode ser uma forma de compensar a baixa luminosidade (Figura 3).

A relação $\mathrm{A} / \mathrm{D}$ é um parâmetro que indica a qualidade de mudas a serem levadas ao campo, uma vez que se espera um equilíbrio no desenvolvimento, entre a parte aérea e o sistema radicular da planta (Campos \& Uchida, 2002). A maior relação $\mathrm{A} / \mathrm{D}$ foi obtida no nível de sombreamento de $50 \%$, a partir dos 124 dias após a emergência. (Figura 4).

Em estudo desenvolvido com a espécie Jacarandá copaia, foi observado os melhores resultados de relação $\mathrm{A} / \mathrm{D}$ aos 173 dias após a repicagem em plantas expostas a 50\% e 70\% de sombreamento (Campos \& Uchida, 2002). Maiores valores de relação $\mathrm{A} / \mathrm{D}$ implicam em plantas menos resistentes no campo às condiçôes impostas pelos fatores ambientais. Foi observado durante o período de avaliação, que a partir dos 82 dias após a emergência, houve uma tendência de diminuição da relação altura/diâmetro. Essa é uma característica importante, para o sucesso de adaptação da planta no campo, pois quanto menor essa relação, mais resistentes são as plantas às condições do ambiente, em decorrência do maior equilíbrio entre as partes da planta.

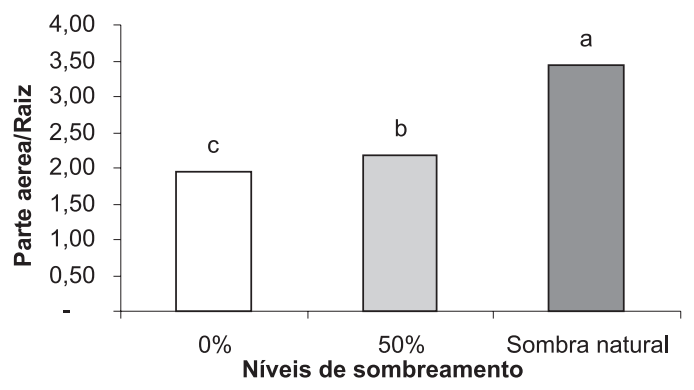

Figura 3 - Razão parte aérea/sistema radicular (PA/R) de plantas de Theobroma grandiflorum (Willd. Ex Spreng.) Schum aos 145 dias, submetidas em três níveis de sombreamento, Gurupi - T0, 2006. 


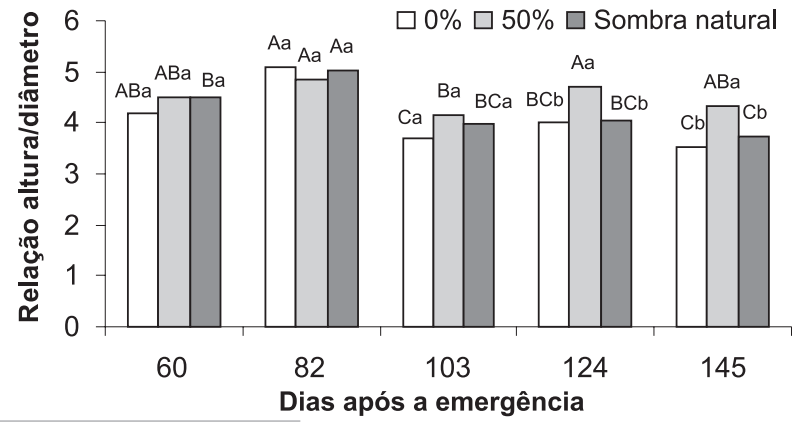

Figura 4 - Relação altura/diâmetro do colo (A/D) em plantas de Theobroma grandiflorum (Willd. Ex Spreng.) Schum, submetida em três níveis de sombreamento, Gurupi - T0, 2006. Médias seguidas pelas mesmas letras maiúsculas considerando DAE e letras minúsculas considerando os níveis de luz não diferem estatisticamente entre si, a $5 \%$ de probabilidade pelo teste tukey.

\section{CONCLUSÃO}

A condição de $50 \%$ de sombreamento pode ser recomendada para a formação de mudas de Theobroma grandiflorum (Willd. Ex Spreng.) Schum, devido o seu melhor desempenho em altura, diâmetro, número de folhas e alocação de massa nas partes da planta.

\section{BIBLIOGRAFIA CITADA}

Barbosa, A.P. 1985. Ecofisiologia do crescimento inicial de mudas de Morototó (Scheflera morototoni, Aubl.Frondin Araliaceae) cultivadas sobre niveis de radiação solar e três niveis de espaçamento. Dissertação de Mestrado, Universidade Federal do Amazonas. 95pp.

Bazzaz, F.A. 1979. The physiological ecology of plant succession. Annual Review of Ecology and Systematics, 10: 351-371.

Campos, M.A.S.; Uchida, T. 2002. Influência do sombreamento no crescimento de mudas de três espécies amazônicas. Pesquisa Agropecuária Brasileira, 37(3): 281-288.

Castro, E.M.; Alvarenga, A.A.; Gomide, M.B. 1996. Crescimento e distribuição de matéria seca de mudas de calabura (Muntingia calabura L.) submetidas a três diferentes níveis de irradiância. Ciência e Agrotecnologia, 20(3): 357-365.

Dias-filho, M.B. 1997. Physiological response of Solanum crinitum Lam. to contrasting light enviroments. Pesquisa Agropecuária Brasileira, 32(8): 789-796.
Felfilli, J.M.; Hilgbert, L.F.; Franco, A.C.; Sousa-Silva, J.C.; Rezende, A.V.; Nogueira, M.V.P. 1999. Comportamento de plântulas de Sclerolobium paniculatum Vog. var. rubiginosum (Tul.) Benth. sob diferentes níveis de sombreamento, em viveiro. Revista Brasileira de Botânica, 22(2): 297-301.

Ferreira, C.A.G. 2000. Recuperação de áreas degradadas. Informe Agropecuário, 21(202): 127-130.

Groninger, J.W.; Seiler, J.R.; Peterson, J.A.; Kreh, R.E. 1996. Growth and photosynthetic responses of four Virginia Piedmont tree species to shade. Tree Physiology, 16: 773-778.

Lorenzi, H. 2000. Arvores Brasileiras: Manual de identificação e cultivo de plantas arbóreas nativas do Brasil. Nova Odessa: $3^{\circ}$ Edição, São Paulo. 368pp.

Mazzei, L.J.; Felfili, J.M.; Rezende, A.V.; Franco, A.C.; Sousa-Silva, J.C. 1998.Crescimento de plântulas de Schefflera morototoni (Aubl.) Maguire, Steyermark e Frodin em diferentes níveis de sombreamento no viveiro. Boletim do Herbário Ezechias Paulo Heringer, 3: 27-36.

Moraes Neto, S.P.; Gonçalves, J.L. de M.; Takaki, M.; Cenci, S.; Gonçalves, J.C. 2000. Crescimento de mudas de algumas espécies arbóreas que ocorrem na mata atlântica, em função do nível de luminosidade. Revista Árvore, 24(1): 35-45.

Muller, C.H.; Figueiredo, F.J.C.; Nascimento, W.M.O.; Galvão, E.U.P.; Stein, R.L.B.; Silva, A.B.; Rodrigues, E.E.L.F.; Carvalho, J.E.U.; Nunes, A.M.L.; Nazaré, R.F.B.; Barbosa, W.C. 1995. A cultura do cupuaçu. Brasília: EMBRAPA-SPI. 61pp.

Murray, D.B., Nichols, F. 1966. Light, shade and growth in some tropical plants. In: light an ecological factor. New iork: Blackwell, p. 249-263.

Pinto, A.M.; Varela, V.P.; Batalha, L.F.P. 1993. Influência do sombreamento no desenvolvimento de mudas de louro pirarucu (Licaria canella (Meissm) Korsterm.). Acta Amazônica, 23(4): 397-404.

Scalon, S.P.Q.; Mussury, R.M.; Rigoni, M.R.; Veraldo, F. 2002. Crescimento inicial de mudas de espécies florestais nativas sob diferentes níveis de sombreamento. Revista Árvore, 26(1): 1-5.

Scalon, S.P.Q.; Mussury, R.M.; Rigoni, M.R.; Filho, H.S. 2003. Crescimento inicial de mudas de Bombacopsis glabra (Pasq.) A. Robins sob condição de sombreamento. Revista Árvore, 27.

Recebido em 22/12/2006

Aceito em 24/07/2007 